\title{
Charmonium description from a generalized screened potential model
}

\author{
P. González \\ Departamento de Física Teórica -IFIC \\ Universitat de Valncia-CSIC \\ E-46100 Burjassot (Valencia), Spain. \\ (E-mail: pedro.gonzalez@uv.es)
}

\begin{abstract}
A generalized screened potential model (GSPM), recently developed to study the bottomonium spectrum, is applied to the calculation of charmonium masses and electromagnetic widths. The presence in the GSPM of more quark-antiquark bound states than in conventional non screened potential models, allows for the assignment of GSPM states to cataloged non conventional $J^{++}$charmonium resonances as well as for the prediction of new (non cataloged) $J^{++}$states. The results obtained seem to indicate that a reasonable overall description of $J^{++}$charmonium resonances is feasible.
\end{abstract}

Keywords: quark, meson, potential 


\section{Introduction}

In a recent paper [1] a new non relativistic quark model for the description of heavy quark mesons has been developed. The novelty of the model, called Generalized Screened Potential Model or GSPM, is the consideration of a lattice motivated quark-antiquark interaction that implicitly incorporates color screening effects from meson-meson configurations. When applied to bottomonium a good spectral description of well established resonances is obtained and a richer high energy spectrum (bigger number of bound states) than the one resulting from the non-screened Cornell potential is predicted. However, the current lack of data does not allow to validate or refute this prediction. In this regard the application of the model to charmonium could be determinant since a plethora of additional states, not fitting into the conventional non-screened Cornell potential framework, has been discovered in the last ten years (see [2, 3, 4] and references therein).

In this article we apply the GSPM to charmonium. We extend the observable analysis beyond the spectral masses to electromagnetic widths, for the model is suitable for their calculation and there exist data to be compared with. We do not analyze strong decays since a fully consistent treatment of them within the GSPM framework (involving the description of mesons containing light quarks) is a formidable task outside the scope of the present study.

We show that a reasonable description of well established and candidates to $\mathrm{J}^{++}$ resonances is feasible. Moreover the model allows for some definite predictions about new resonances what might be used in future experimental searches to further check its validity. Regarding $1^{--}$states the presence of overlapping thresholds limits the applicability of the GSPM to spectral energies quite below the first meson-meson threshold becoming then completely equivalent to the Cornell model.

The article contents are organized as follows. In Section 2 a brief review of the GSPM is presented. In Section 3 the model is applied to the calculation of the charmonium spectrum and electromagnetic widths and the results are compared to data. A calculation from a non screened Cornell potential is also shown for comparison. Finally, in Section 4 our main results and conclusions are summarized.

\section{Generalized Screened Potential Model (GSPM)}

The Generalized Screened Potential Model (GSPM) is based on the assumption that a heavy quark meson description can be attained from the consideration of effective valence quark degrees of freedom interacting through a potential that incorporates screening effects from meson-meson configurations.

More precisely, the Generalized Screened Potential that we shall call $V(r)$ henceforth tries to implement within a quark model framework the lattice results for the energy of two static color sources (heavy quark and heavy antiquark) in terms of their distance, $E_{\text {lattice }}(r)$, when the mixing of the quenched quark-antiquark configuration with open flavor meson-meson ones is taken into account. In reference [5] the lattice calculation 
for the case of one open flavor meson-meson configuration was performed, the resulting $E_{\text {lattice }}(r)$ having a different form below and above the meson-meson threshold. For the two threshold case an educated guess for $E_{\text {lattice }}(r)$ was done (see Fig. 22 in [5]). A simplified generalization of these lattice results to the many threshold case was proposed in reference [1]. From it a static quark-antiquark potential, $V(r)$, was derived by means of a Born-Oppenheimer approximation, say by subtracting the quark and antiquark masses, $m_{Q}$ and $m_{\bar{Q}}$, from the static energy.

Explicitly, by calling $M_{T_{i}}$ with $i \geq 1$ the masses of the physical meson-meson thresholds, $T_{i}$, with a given set of quantum numbers $I\left(J^{P C}\right)$, and defining $M_{T_{0}} \equiv 0$ for a unified notation (note that $T_{0}$ does not correspond to any physical meson-meson threshold), the form of $V(r)$ in the different energy regions (specified as energy interval subindices) reads:

$$
V_{\left[M_{T_{0}}, M_{T_{1}}\right]}(r)= \begin{cases}\sigma r-\frac{\chi}{r} & r \leq r_{T_{1}} \\ M_{T_{1}}-m_{Q}-m_{\bar{Q}} & r \geq r_{T_{1}}\end{cases}
$$

and

$$
V_{\left[M_{\left.T_{j-1}, M_{T_{j}}\right]}\right.}(r)=\left\{\begin{array}{lr}
M_{T_{j-1}}-m_{Q}-m_{\bar{Q}} & r \leq r_{T_{j-1}} \\
\sigma r-\frac{\chi}{r} & r_{T_{j-1}} \leq r \leq r_{T_{j}} \\
M_{T_{j}}-m_{Q}-m_{\bar{Q}} & r \geq r_{T_{j}}
\end{array}\right.
$$

for $j>1$, with the crossing radii $r_{T_{i}}(i \geq 1)$ defined by

$$
\sigma r_{T_{i}}-\frac{\chi}{r_{T_{i}}}=M_{T_{i}}-m_{Q}-m_{\bar{Q}}
$$

with the parameters $\sigma$ and $\chi$ standing for the string tension and the color coulomb strength respectively.

Thus $V(r)$ has in each energy region between neighbor thresholds a Cornell form but modulated at short and long distances by these thresholds.

Thus for example in Fig. 1 the form of $V(r)$ in the first and second energy regions is drawn for $c \bar{c}$ states with $I^{G}\left(J^{P C}\right)=0^{+}\left(1^{++}\right)$quantum numbers, whose first threshold $T_{1}$ corresponds to $D \overline{D^{*}}$ and its second threshold $T_{2}$ to $D_{s}^{+} D_{s}^{*-}$.

Let us remark that $V(r)$ is an energy dependent potential in the sense that its form differs in the different energy regions delimited by the thresholds. Actually this is the essential difference with other screened potential models which have been also employed for the description of charmonium [6, 7].

It is also important to emphasize that in any energy region the potential is strictly confining in the sense that only bound states are obtained as solutions of the Schrödinger equation in such energy region (see next Section). 


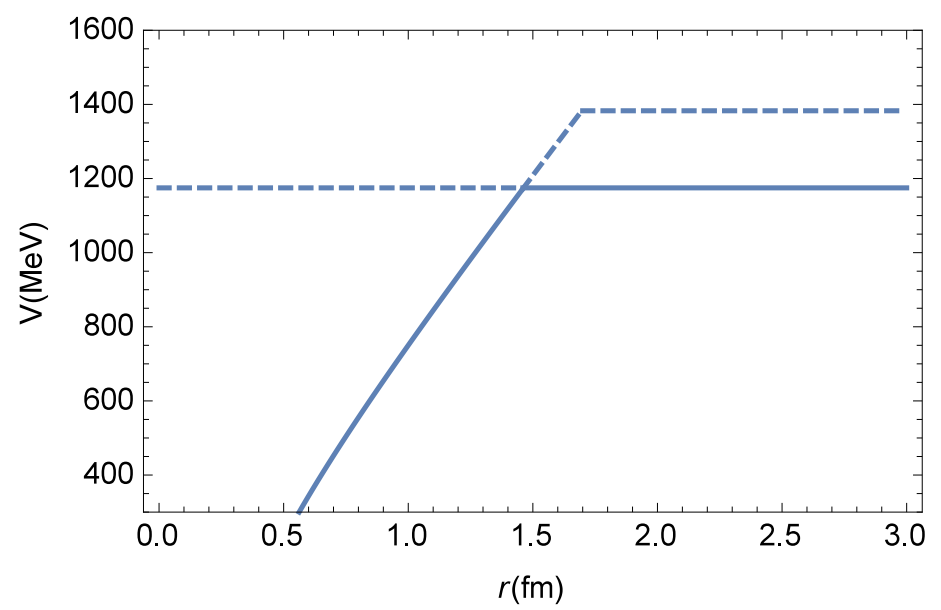

Figure 1: Generalized screened potential $V(r)$. The solid (dashed) line indicates the potential in the first (second) energy region for $0^{+}\left(1^{++}\right) c \bar{c}$ states with $m_{c}=1348.6$ $\mathrm{MeV}, \sigma=850 \mathrm{MeV} / \mathrm{fm}, \chi=100 \mathrm{MeV} . \mathrm{fm}, M_{T_{1}}=3872 \mathrm{MeV}$ and $M_{T_{2}}=4080 \mathrm{MeV}$ (values of the parameters and threshold masses from Section 3).

\section{Charmonium}

From the defined $V(r)$, charmonium states in the energy region $\left[M_{T_{i-1}}, M_{T_{i}}\right]$, characterized by a definite set of quantum numbers $I^{G}\left(J^{P C}\right)$, are obtained by solving the Schrödinger equation for $V_{\left[M_{T_{i-1}}, M_{T_{i}}\right]}(r)$.

In order to get the solutions we previously fix the values of the parameters of the model and list the open charm meson-meson threshold masses to be considered. Then we detail the calculation of the spectrum for a particular case before giving the general results. Next we assign calculated states to experimental resonances and use the corresponding wave functions to evaluate electromagnetic widths.

\subsection{Parameters}

Let us realize that for energies quite below the first corresponding thresholds the potential $V(r)$ is almost completely equivalent to a Cornell one:

$$
V_{\text {Cor }}(r) \equiv \sigma r-\frac{\chi}{r} \quad(r: 0 \rightarrow \infty)
$$

But the use of the conventional Cornell potential teaches us that the charm-anticharm $(c \bar{c})$ system may be a relativistic one [9]. This makes debatable the application of the GSPM to charmonium. In the spirit of quark model calculations we shall assume that the effectiveness of the parameters (quark mass, string tension and coulomb strength) may be appropriately taking into account, at least in part, relativistic corrections. We can also 
invoke the effectiveness of the parameters regarding additional contributions from light quark-antiquark pairs apart from the implicitly considered meson-meson configurations.

Let us also note that $i$ ) no threshold widths have been considered and $i i$ ) the accumulative interacting effect from different meson-meson configurations with the same threshold mass has not been implemented. (Indeed if a meson-meson configuration gives rise to a screening of the quark and antiquark color charges for a given energy then a reinforcement of the screening is expected when more meson-meson configurations with the same threshold mass are available.)

Hence we shall restrict the application of the model to those energy regions involving non degenerate, isolated (in the sense of not having any significant experimental overlap due to their widths) thresholds.

Even so the model may be too simplistic for an accurate description of real mesons. On the one hand $V(r)$ does not contain spin dependent terms that we know may give significant contributions to the masses of the lower spectral states (see for example [10]). On the other hand the effect of any threshold has been approximated by an abrupt (instead of a physically soft) change in the potential at the crossing radii. Moreover $S U$ (3) flavor symmetry has been considered when the same effect (flattening of the potential from the crossing radii) from thresholds with $s \bar{s}, u \bar{u}$ or $d \bar{d}$ content has been implemented despite the fact that the probability of formation for each of these pairs may be different.

Keeping in mind these possible shortcomings we shall try to show that such a simple model could provide us with some insight onto the dominant dynamic mechanisms governing the charmonium structure.

As we are dealing with a spin independent potential we shall compare as usual the calculated $s$ - wave state masses with spin-triplet data, the $p$ - wave state masses with the centroids obtained from data and the $d$ - wave states with the few existing experimental candidates.

Aiming at a joint description of charmonium and bottomonium we shall use for both the same values for the parameters of the potential. From [1] we have $\sigma=850 \mathrm{MeV} / \mathrm{fm}$ and $\chi=100 \mathrm{MeV}$.fm. Let us realize that this string tension value $\sqrt{\sigma}=410 \mathrm{MeV}$ is within the interval usually accepted for it from phenomenology (see for instance [11]). As for the Coulomb strength $\chi$ its value corresponds to a strong quark-gluon-quark coupling $\alpha_{s}=\frac{3 \chi}{4 \hbar} \simeq 0.38$, in agreement with the value derived from QCD from the fine structure splitting of $1 p$ states in charmonium [12]. Regarding the remaining parameter of the model $m_{c}$ we fix its value to get a reasonable overall fit to the spectrum.

Thus the set of parameters that will be used henceforth is

$$
\begin{gathered}
\sigma=850 \mathrm{MeV} / \mathrm{fm} \\
\chi=100 \mathrm{MeV} . \mathrm{fm} \\
m_{c}=1348.6 \mathrm{MeV}
\end{gathered}
$$

where the value of the charm mass $m_{c}=1348.6 \mathrm{MeV}$ has been fine tuned to fit the mass 
of the well established non conventional charmonium state $X(3872)$ since this resonance may be naturally described in the GSPM as explained later on.

It is important to remark that due to the effective character of the parameters a better overall spectral fit (differences from calculated masses to data of $35 \mathrm{MeV}$ at most) could be achieved by choosing for example $\sigma=750 \mathrm{MeV} / \mathrm{fm}, \chi=100 \mathrm{MeV}$.fm and $m_{c}=1407.8 \mathrm{MeV}$. However as this new fit makes no difference at all in the resulting number of spectral states we prefer to maintain the same potential description as in bottomonium.

\section{$3.2 \quad I\left(J^{P C}\right)$ Thresholds}

In order to apply the GSPM to a particular set of charmonium $(I=0)$ states with definite $J^{P C}$ we need the masses $M_{T_{i}}$ for open charm meson - meson thresholds coupling to these quantum numbers. From these masses the crossing radii $r_{T_{i}}$ are immediately calculated from (3).

Let us realize that the static approach we follow to build the potential implies that the heavy quark and antiquark or, quite equivalently, the two charmed mesons forming the threshold, are in a relative $S$ - wave so that the threshold mass is just the sum of the masses of the mesons.

The list of known thresholds, their masses and the corresponding crossing radii appear in Tables 1 and 2 where a simplified notation has been used: a threshold has been denoted by the first meson-meson component entering in the $I\left(J^{P C}\right)$ linear combination. Thus, the first $0\left(1^{++}\right)$threshold in Table 1, $D^{0}{\overline{D^{*}}}^{0}(2007)$ denotes $\left(D^{0}{\overline{D^{*}}}^{0}(2007), D^{+} D^{*}(2010)^{-}\right)_{I=0, J^{P}=1^{+}}+$c.c. where c.c. stands for the charge conjugate.

We have used isospin symmetry to construct thresholds with well defined isospin. This means that we are neglecting the mass differences between the electrically neutral and charged members of the same isospin multiplet, for example $D^{0}$ and $D^{ \pm}$with PDG quoted masses [2] 1864.91 \pm 0.17 and $1869.5 \pm 0.4$ respectively or $D^{*}(2007)^{0}$ and $D^{*}(2010)^{-}$ with quoted masses $2006.98 \pm 0.15$ and $2010.21 \pm 0.13$ respectively. For the calculation of the threshold masses we have used the lower mass value in any isospin multiplet (1865 $\mathrm{MeV}$ and $2007 \mathrm{MeV}$ in the examples just mentioned).

Regarding the $C$ parity, for a threshold formed by two mesons $\mathfrak{M}_{1}$ and $\mathfrak{M}_{2}$ we can construct the combinations $\left(\mathfrak{M}_{1} \mathfrak{M}_{2} \pm\right.$ c.c. $)$ with $C$ parity + and - respectively. Notice though that if $\mathfrak{M}_{2}=\overline{\mathfrak{M}_{1}}$ then, as the two mesons are in $S-$ wave, we have $\overline{\mathfrak{M}_{1}} \mathfrak{M}_{1}=$ $(-)^{j_{1}+j_{1}-j} \mathfrak{M}_{1} \overline{\mathfrak{M}_{1}}$ where $j_{1}$ stands for the spin of $\mathfrak{M}_{1}$ and $j$ for the total spin of the threshold. Therefore only one combination in $\mathfrak{M}_{1} \overline{\mathfrak{M}_{1}} \pm$ c.c. is allowed for a given value of $j$ (the other vanishes). For example the $I=0$ threshold $D^{*} \overline{D^{*}}$ with $j_{1}=1$ has only positive $C$ parity when coupled to $j=0,2$ (and only negative $C$ parity when coupled to $j=1)$. 


\begin{tabular}{|c|c|c|c|c|c|}
\hline$I\left(J^{P C}\right)$ & $T_{i}$ & Charmonium Thresholds & $\left(J_{1}^{P}, J_{2}^{P}\right)$ & $\begin{array}{c}M_{T_{i}} \\
(\mathrm{MeV})\end{array}$ & $\begin{array}{c}r_{T_{i}} \\
(\mathrm{fm})\end{array}$ \\
\hline \multicolumn{6}{|l|}{$0\left(0^{++}\right)$} \\
\hline & $T_{1}$ & $D^{0} \bar{D}^{0}$ & $\left(0^{-}, 0^{-}\right)$ & 3730 & 1.31 \\
\hline & $T_{2}$ & $D_{s}^{+} D_{s}^{-}$ & $\left(0^{-}, 0^{-}\right)$ & 3937 & 1.54 \\
\hline & $T_{3}$ & $D^{* 0}(2007){\overline{D^{*}}}^{0}(2007)$ & $\left(1^{-}, 1^{-}\right)$ & 4014 & 1.62 \\
\hline & $T_{4}$ & $D_{s}^{*+} D_{s}^{*-}$ & $\left(1^{-}, 1^{-}\right)$ & 4224 & 1.86 \\
\hline & $T_{5}$ & $D^{0} \bar{D}^{0}(2550)$ & $\left(0^{-}, 0^{-}\right)$ & 4405 & 2.07 \\
\hline \multicolumn{6}{|l|}{$0\left(1^{++}\right)$} \\
\hline & $T_{1}$ & $D^{0}{\overline{D^{*}}}^{0}(2007)$ & $\left(0^{-}, 1^{-}\right)$ & 3872 & 1.46 \\
\hline & $T_{2}$ & $D_{s}^{+} D_{s}^{*-}$ & $\left(0^{-}, 1^{-}\right)$ & 4080 & 1.70 \\
\hline \multicolumn{6}{|l|}{$0\left(2^{++}\right)$} \\
\hline & $T_{1}$ & $D^{*}(2007)^{0}{\overline{D^{*}}}^{0}(2007)$ & $\left(1^{-}, 1^{-}\right)$ & 4014 & 1.62 \\
\hline & $T_{2}$ & $D_{s}^{*+} D_{s}^{*-}$ & $\left(1^{-}, 1^{-}\right)$ & 4224 & 1.86 \\
\hline
\end{tabular}

Table 1: Open charm meson-meson thresholds for $0\left(J^{++}\right)$charmonium states. $J_{1}^{P}$ and $J_{2}^{P}$ stand for the angular momenta of the mesons forming the threshold. Threshold masses $\left(M_{T_{i}}\right)$ obtained from the charmed and charmed strange meson masses quoted in [2]. Crossing radii $\left(r_{T_{i}}\right)$ calculated from (3). 


\begin{tabular}{|c|c|c|c|c|c|}
\hline \multirow{9}{*}{$\begin{array}{l}I\left(J^{P C}\right) \\
0\left(1^{--}\right)\end{array}$} & $T_{i}$ & Charmonium Thresholds & $\left(J_{1}^{P}, J_{2}^{P}\right)$ & $\begin{array}{c}M_{T_{i}} \\
(\mathrm{MeV})\end{array}$ & $\begin{array}{c}r_{T_{i}} \\
(\mathrm{fm})\end{array}$ \\
\hline & $T_{1}$ & $\begin{array}{l}D^{0}{\overline{\bar{D}_{1}}}^{0}(2420) \\
D^{0}{\overline{D_{1}}}^{0}(2430)\end{array}$ & $\left(0^{-}, 1^{+}\right)$ & 4287 & 1.93 \\
\hline & $T_{2}$ & $D^{*}(2007)^{0}{\overline{D_{0}^{*}}}^{0}(2400)$ & $\left(1^{-}, 0^{+}\right)$ & 4325 & 1.98 \\
\hline & $T_{3}$ & $\begin{array}{c}D^{*}(2007)^{0}{\overline{D_{1}}}^{0}(2420) \\
D^{*}(2007)^{0}{\overline{D_{1}}}^{0}(2430) \\
D_{s}^{+} D_{s 1}(2460)^{-} \\
D_{s}^{*+} D_{s 0}^{*}(2317)^{-}\end{array}$ & $\begin{array}{l}\left(1^{-}, 1^{+}\right) \\
\left(1^{-}, 1^{+}\right) \\
\left(0^{-}, 1^{+}\right) \\
\left(1^{-}, 0^{+}\right)\end{array}$ & 4429 & 2.09 \\
\hline & $T_{4}$ & $D^{*}(2007)^{0}{\overline{D_{2}^{*}}}^{0}(2460)$ & $\left(1^{-}, 2^{+}\right)$ & 4470 & 2.14 \\
\hline & $T_{5}$ & $D_{s}^{+} D_{s 1}(2536)^{-}$ & $\left(0^{-}, 1^{+}\right)$ & 4504 & 2.18 \\
\hline & $T_{6}$ & $D_{s}^{*+} D_{s 1}(2460)^{-}$ & $\left(1^{-}, 1^{+}\right)$ & 4572 & 2.26 \\
\hline & $T_{7}$ & $D_{s}^{*+} D_{s 1}(2536)^{-}$ & $\left(1^{-}, 1^{+}\right)$ & 4648 & 2.35 \\
\hline & $T_{8}$ & $D_{s}^{*+} D_{s 2}^{*}(2573)^{-}$ & $\left(1^{-}, 2^{+}\right)$ & 4685 & 2.39 \\
\hline
\end{tabular}

Table 2: Open charm meson-meson thresholds for $0\left(1^{--}\right)$charmonium states. $J_{1}^{P}$ and $J_{2}^{P}$ stand for the angular momenta of the mesons forming the threshold. Threshold masses $\left(M_{T_{i}}\right)$ obtained from the charmed and charmed strange meson masses quoted in [2]. Crossing radii $\left(r_{T_{i}}\right)$ calculated from (33). 
A look at Table 1 makes clear that for $0\left(J^{++}\right)$states only non degenerate isolated thresholds are present. Therefore the GSPM can be safely applied. On the contrary for $0\left(1^{--}\right)$states, Table 2, there are degenerate $\left(T_{1}\right.$ and $\left.T_{3}\right)$ and overlapping thresholds (for instance $T_{1}$ and $T_{3}$ overlap with $T_{2}$ due to the large width $(267 \mathrm{MeV})$ of $\left.\overline{D_{0}^{*}}(2400)\right)$. Therefore, at its present stage the GSPM can only be consistently applied to $0\left(1^{--}\right)$ states quite below the first threshold. In consequence we shall restrict our study in this case to the first energy region.

\section{$3.3 \quad$ Spectrum}

Charmonium $(c \bar{c})$ states are obtained by solving the Schrödinger equation for $V(r)$. As in any energy region it is a radial potential we use spectroscopic notation $k \equiv n l$, in terms of the radial, $n$, and orbital angular momentum, $l$, quantum numbers, to denote its bound states. Thus in the energy region $\left[M_{T_{i-1}}, M_{T_{i}}\right]$ we have

$$
\begin{aligned}
& \left(\mathcal{T}+V_{\left[M_{T_{i-1}}, M_{T_{i}}\right]}\right)\left|(c \bar{c})_{\left.k_{\left[T_{i-1}, T_{i}\right]}\right]}\right\rangle \\
& =M_{\left.k_{\left[T_{i-1}, T_{i}\right]}\right]}\left|(c \bar{c})_{\left.k_{\left[T_{i-1}, T_{i}\right]}\right\rangle}\right\rangle
\end{aligned}
$$

where $\mathcal{T}$ stands for the kinetic energy operator, $\left|(c \bar{c})_{k_{\left[T_{i-1}, T_{i}\right]}}\right\rangle$ for the bound states and $M_{k_{\left[T_{i-1}, T_{i}\right]}}$ for their masses.

Let us consider for example the $0^{+}\left(1^{++}\right)$spectral states. In the first energy region the potential $V(r)$ has the form $V_{\left[M_{T_{0}}, M_{T_{1}}\right]}(r)$, given by (1) (solid line in Fig. 1)

$$
V_{[0,3872]}(r)=\left\{\begin{array}{cc}
\sigma r-\frac{\chi}{r} & r \leq 1.46 \mathrm{fm} \\
1174.8 \mathrm{MeV} & r \geq 1.46 \mathrm{fm}
\end{array}\right.
$$

where $M_{T_{1}}$ and $r_{T_{1}}$ have been taken from Table 1 and the values of the parameters $\left(\sigma, \chi, m_{c}\right)$ are given by (5).

By solving the Schrödinger equation for $V_{[0,3872]}(r)$ we get the GSPM $0^{+}\left(1^{++}\right)$spectrum in the first energy region $\left[M_{T_{0}}=0, M_{T_{1}}=3872 \mathrm{MeV}\right]$. It has two bound states states, $1 p_{\left[T_{0}, T_{1}\right]}$ and $2 p_{\left[T_{0}, T_{1}\right]}$, whose masses $M_{k_{\left[T_{0}, T_{1}\right]}}$ generically denoted by $M_{G S P M}$ are listed in Table 3 .

In the second energy region, $\left[M_{T_{1}}=3872 \mathrm{MeV}, M_{T_{2}}=4080 \mathrm{MeV}\right]$, the potential $V(r)$ has the form $V_{\left[M_{T_{1}}, M_{T_{2}}\right]}(r)$, given by (2) (dashed line in Fig. 1):

$$
V_{[3872,4080]}(r)=\left\{\begin{array}{cc}
1174.8 \mathrm{MeV} & r \leq 1.46 \mathrm{fm} \\
\sigma r-\frac{\chi}{r} & 1.46 \mathrm{fm} \leq r \leq 1.70 \mathrm{fm} \\
1382.8 \mathrm{MeV} & r \geq 1.70 \mathrm{fm}
\end{array}\right.
$$




$$
\begin{aligned}
& \begin{array}{ccccc}
c \bar{c} & & & & \\
0\left(1^{++}\right) & {\left[T_{i-1}, T_{i}\right]} & {\left[M_{T_{i-1}}, M_{T_{i}}\right]} & \text { GSPM } & M_{G S P M} \\
& \mathrm{MeV} & \text { States } & \mathrm{MeV} \\
& & k_{\left[T_{i-1}, T_{i}\right]} & \\
\hline
\end{array} \\
& \begin{array}{lll}
{\left[T_{0}, T_{1}\right] \quad[0,3872]} & 1 p_{\left[T_{0}, T_{1}\right]} & 3456.1 \\
& 2 p_{\left[T_{0}, T_{1}\right]} & 3871.7
\end{array} \\
& {\left[T_{1}, T_{2}\right] \quad[3872,4080] \quad 1 p_{\left[T_{1}, T_{2}\right]} \quad 4017.3}
\end{aligned}
$$

Table 3: Calculated $0^{+}\left(1^{++}\right)$charmonium masses from $V(r)$, generically denoted by $M_{G S P M}$, in the first two energy regions specified by the thresholds $\left[T_{i-1}, T_{i}\right]$ and their masses $\left[M_{T_{i-1}}, M_{T_{i}}\right]$.

where the threshold masses and crossing radii are taken from Table 11. The spectrum has only one bound state $1 p_{\left[T_{1}, T_{2}\right]}$ whose mass $M_{1 p_{\left[T_{1}, T_{2}\right]}}$ generically denoted by $M_{G S P M}$ is listed in Table 3 .

By proceeding in the same way for higher energy regions and for different quantum numbers we get the complete GSPM bound state spectrum. But before listing the calculated spectral masses it may be illustrative to analyze the effect produced by just one threshold. For this purpose we shall compare the results obtained from the Cornell potential with the ones obtained from a GSPM with only one threshold.

Let us consider again $0^{+}\left(1^{++}\right)$states and calculate the spectrum if only the threshold $T_{1}$ (corresponding to $D^{0}{\overline{D^{*}}}^{0}(2007)$ ) is present. Then there will be two energy regions. In the first one, $\left[M_{T_{0}}=0, M_{T_{1}}=3872 \mathrm{MeV}\right]$, the potential is given by (7). Hence there are two bound states, $1 p_{\left[T_{0}, T_{1}\right]}$ and $2 p_{\left[T_{0}, T_{1}\right]}$, with the masses previously calculated (see Table 3) which have been listed again in Table 4. In the second energy region, $\left[M_{T_{1}}=3872 \mathrm{MeV}, \infty[\right.$, the potential reads

$$
V_{[3872, \infty[}(r)= \begin{cases}1174.8 \mathrm{MeV} & r \leq 1.46 \mathrm{fm} \\ \sigma r-\frac{\chi}{r} & r \geq 1.46 \mathrm{fm}\end{cases}
$$

and has an infinite number of bound states. The masses of the two lowest states in this energy region are listed in Table 4 .

For the sake of comparison we calculate the Cornell spectrum in the same energy interval (from 0 to $4400 \mathrm{MeV}$ ) from the same values of the parameters $\sigma, \chi$ and $m_{c}$ 


\begin{tabular}{cc|cc}
$\begin{array}{c}\text { GSPM } \\
\text { States }\end{array}$ & $\begin{array}{c}M_{G S P M} \\
\mathrm{MeV}\end{array}$ & $\begin{array}{c}M_{\text {Cor }} \\
\mathrm{MeV}\end{array}$ & $\begin{array}{c}\text { Cornell } \\
\text { States }\end{array}$ \\
\hline $1 p_{\left[T_{0}, T_{1}\right]}$ & 3456.1 & 3456.2 & $1 p$ \\
$2 p_{\left[T_{0}, T_{1}\right]}$ & 3871.7 & 3910.9 & $2 p$ \\
$1 p_{\left[T_{1}, \infty[\right.}$ & 4029.3 & & \\
$2 p_{\left[T_{1}, \infty[\right.}$ & 4303.3 & 4294.6 & $3 p$
\end{tabular}

Table 4: Calculated $0^{+}\left(1^{++}\right)$charmonium masses up to $4400 \mathrm{MeV}$ when only the first threshold is considered: $M_{G S P M}$. Masses from the Cornell potential, $M_{C o r}$, are also shown for comparison. Conventional spectroscopic notation has been used to denote the Cornell states.

given by (5). The results are also listed in Table 4. We immediately realize that in the spectral region considered there are four GSPM states $\left(1 p_{\left[T_{0}, T_{1}\right]}, 2 p_{\left[T_{0}, T_{1}\right]}, 1 p_{\left[T_{1}, \infty[\right.}, 2 p_{\left[T_{1}, \infty[\right.}\right)$ for only three Cornell states $(1 p, 2 p, 3 p)$. Moreover, the masses of the first and fourth GSPM states are quite the same as the masses of the first and third Cornell states. Since the GSPM potential differs from the Cornell one in the incorporation of the threshold $T_{1} \equiv D^{0}{\overline{D^{*}}}^{0}(2007)$ we may interpret these results by saying that the second $\left(2 p_{\left[T_{0}, T_{1}\right]}\right)$ and third $\left(1 p_{\left[T_{1}, \infty[\right.}\right)$ GSPM states are effectively describing the mixing of the second Cornell (2p) state with the $D^{0}{\overline{D^{*}}}^{0}(2007)$ configuration. Therefore the effect of the threshold is the appearance of one more spectral state (notice though that if the $2 p$ Cornell state were farther above the threshold the GSPM would not generate the $2 p_{\left[T_{0}, T_{1}\right]}$ state).

It may also be interesting to compare the resulting radial wave functions for the $2 p_{\left[T_{0}, T_{1}\right]}$ GSPM state and the $2 p$ Cornell state. This comparison is drawn in Fig. 2.

As can be checked the $2 p_{\left[T_{0}, T_{1}\right]}$ radial wave function extends to much larger distances than the $2 p$ one. If we consider the $2 p_{\left[T_{0}, T_{1}\right]}$ state as an effective description of the experimental $X(3872)$ and the $2 p$ Cornell state as describing a (non experimental) conventional $\chi_{c 1}(2 p)$ state then it is clear the difference between them. The comparison of the respective root square mean radii, $3.6 \mathrm{fm}$ for $X(3872)$ and $1.1 \mathrm{fm}$ for $\chi_{c 1}(2 p)$, indicates the screening of the heavy quark color charges in $X(3872)$ due to the presence of the threshold. (Let us point out that in a couple channel treatment involving quenched quark-antiquark and meson-meson configurations this would correspond to the presence of a $D^{0}{\overrightarrow{D^{*}}}^{0}(2007)$ wave function component.) 


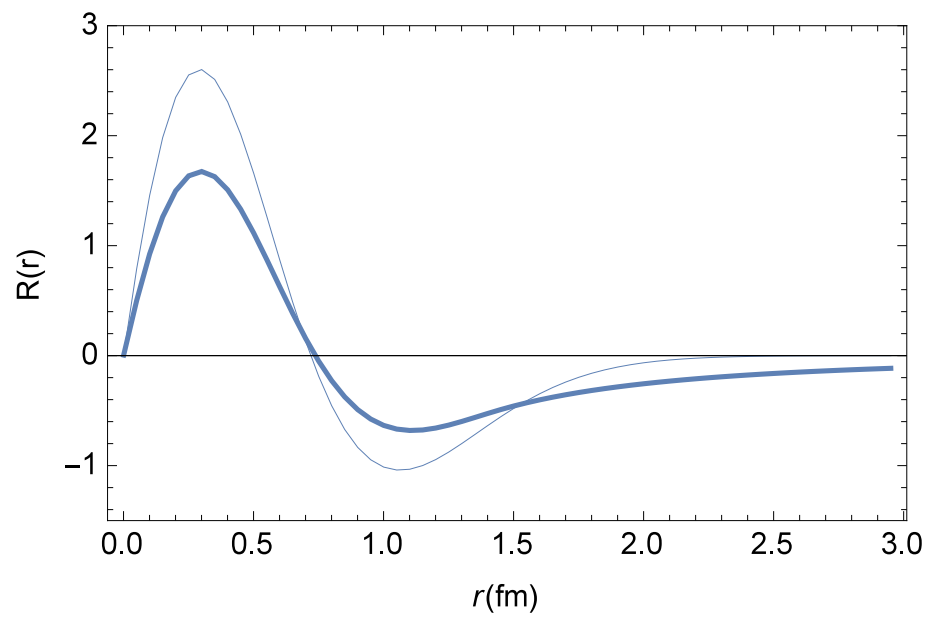

Figure 2: Radial wave functions $\mathrm{R}(\mathrm{r})$ (in units $\left.\mathrm{fm}^{-\frac{3}{2}}\right)$ for the $1^{++}\left(2 p_{\left[T_{0}, T_{1}\right]}\right)$ GSPM state (thick line) and the $1^{++}(2 p)$ Cornell state (thin line).

\subsection{1 $0^{+}\left(J^{++}\right)$GSPM States}

The spectrum for $0^{+}\left(J^{++}\right) c \bar{c}$ states from $V(r)$ is shown in Table 5 , The spectrum from the Cornell potential $V_{C o r}(r)$ given by (4) with the same values of the parameters $\sigma, \chi$ and $m_{c}$ is also listed for comparison.

A glance at the table confirms the presence of a bigger number of GSPM states than Cornell ones even ignoring possible additional $1^{++}$states above $4080 \mathrm{MeV}$ and $2^{++}$states above $4224 \mathrm{MeV}$. More precisely there are (at least) four $J^{++}$GSPM states in the energy interval $4000-4400 \mathrm{MeV}$ for only one Cornell state. Since the calculated masses of three of these GSPM states are in good correspondence with the masses of $X(4140) 0^{+}\left(?^{?+}\right)$ and $X(4350) 0^{+}\left(?^{?+}\right)$, the currently existing experimental candidates to $0^{+}\left(J^{++}\right)$states in that energy interval (see Particle Listing in [2]; see also [3]), a tentative assignment of GSPM states to these candidates has been done in Table 5. From it a guess for their unknown quantum numbers comes out: $X(4140) 0^{+}\left(0^{++}\right)$or $X(4140) 0^{+}\left(2^{++}\right)$and $X(4350) 0^{+}\left(0^{++}\right)$.

Furthermore the model predicts the existence of at least two new $0^{+}\left(\mathrm{J}^{++}\right)$resonances in the energy interval considered. One of them, that we shall call $C(4140)$ ( $C$ standing for theoretical candidate) would be assigned to the $2^{++}\left(1 p_{\left[T_{1}, T_{2}\right]}\right)$ or $0^{++}\left(1 p_{\left[T_{3}, T_{4}\right]}\right)$ GSPM state at $4140.2 \mathrm{MeV}$ (see Table 5). Let us note that the existence of this state in the GSPM is linked to the existence of the $0^{++}\left(1 p_{\left[T_{3}, T_{4}\right]}\right)$ or $2^{++}\left(1 p_{\left[T_{1}, T_{2}\right]}\right)$ state that we have assigned to $X(4140)$; as both states are in between the same thresholds 


\begin{tabular}{|c|c|c|c|c|c|}
\hline$J^{P C}$ & $\begin{array}{c}\text { GSPM } \\
\text { States } \\
k_{\left[T_{i-1}, T_{i}\right]}\end{array}$ & $\begin{array}{c}M_{G S P M} \\
\mathrm{MeV}\end{array}$ & $\begin{array}{c}M_{P D G} \\
\mathrm{MeV}\end{array}$ & $\begin{array}{l}M_{C o r} \\
\mathrm{MeV}\end{array}$ & $\begin{array}{c}\text { Cornell } \\
\text { States } \\
k\end{array}$ \\
\hline $0^{++}$ & $1 p_{\left[T_{0}, T_{1}\right]}$ & 3456.1 & $3414.75 \pm 0.31$ & 3456.2 & $1 p$ \\
\hline $1^{++}$ & $1 p_{\left[T_{0}, T_{1}\right]}$ & 3456.1 & $3510.66 \pm 0.07$ & 3456.2 & $1 p$ \\
\hline $2^{++}$ & $1 p_{\left[T_{0}, T_{1}\right]}$ & 3456.1 & $3556.20 \pm 0.09$ & 3456.2 & $1 p$ \\
\hline $1^{++}$ & $2 p_{\left[T_{0}, T_{1}\right]}$ & 3871.7 & $3871.69 \pm 0.17$ & 3910.9 & $2 p$ \\
\hline $0^{++}$ & $1 p_{\left[T_{1}, T_{2}\right]}$ & 3897.9 & $3918.4 \pm 1.9$ & 3910.9 & $2 p$ \\
\hline $2^{++}$ & $2 p_{\left[T_{0}, T_{1}\right]}$ & 3903.0 & $3927.2 \pm 2.6$ & 3910.9 & $2 p$ \\
\hline $1^{++}$ & $1 p_{\left[T_{1}, T_{2}\right]}$ & 4017.3 & & & \\
\hline $0^{++}$ & $1 p_{\left[T_{3}, T_{4}\right]}$ & 4140.2 & & & \\
\hline & & & $X(4140)$ & & \\
\hline $2^{++}$ & $1 p_{\left[T_{1}, T_{2}\right]}$ & 4140.2 & & & \\
\hline $0^{++}$ & $1 p_{\left[T_{4}, T_{5}\right]}$ & 4325.1 & $X(4350)$ & 4294.6 & $3 p$ \\
\hline
\end{tabular}

Table 5: Calculated $J^{++}$charmonium masses from $V(r): M_{G S P M}$ up to $4350 \mathrm{MeV}$. The $0^{++}\left(1 p_{\left[T_{2}, T_{3}\right]}\right)$ row has been omitted since there is no GSPM bound state in that energy region. For $1^{++}$we do not list any state above $4080 \mathrm{MeV}$ due to the current incomplete knowledge about thresholds above this energy. The same for $2^{++}$states above 4224 $\mathrm{MeV}$. Masses for experimental resonances, $M_{P D G}$, have been taken from [2] (when a resonance appears in the Particle Listing section of [2] but not in the Summary Table we write the name of the resonance that contains the nominal mass between parenthesis). For $p$ waves we quote separately the $n p_{0}, n p_{1}$ and $n p_{2}$ states. Masses from the Cornell potential, $M_{C o r}$, are also shown for comparison. 


\begin{tabular}{cccccc}
$J^{P C}$ & $\begin{array}{c}\text { GSPM } \\
\text { States }\end{array}$ & $\begin{array}{c}M_{G S P M} \\
\mathrm{MeV}\end{array}$ & $\begin{array}{c}M_{P D G} \\
\mathrm{MeV}\end{array}$ & $\begin{array}{c}M_{\text {Cor }} \\
\mathrm{MeV}\end{array}$ & $\begin{array}{c}\text { Cornell } \\
\text { States } \\
k\end{array}$ \\
\hline$k_{\left[T_{i-1}, T_{i}\right]}$ & & & & & \\
$1^{--}$ & $1 s_{\left[T_{0}, T_{1}\right]}$ & 3046.0 & $3096.916 \pm 0.011$ & 3046.0 & $1 s$ \\
& $2 s_{\left[T_{0}, T_{1}\right]}$ & 3632.2 & $3686.09 \pm 0.04$ & 3632.2 & $2 s$ \\
$1 d_{\left[T_{0}, T_{1}\right]}$ & 3743.5 & $3773.15 \pm 0.33$ & 3743.5 & $1 d$ \\
$3 s_{\left[T_{0}, T_{1}\right]}$ & 4063.2 & $4039 \pm 1$ & 4065.8 & $3 s$ \\
$2 d_{\left[T_{0}, T_{1}\right]}$ & 4139.3 & $4191 \pm 5$ & 4142.8 & $2 d$
\end{tabular}

Table 6: Calculated $1^{--}$charmonium masses from $V(r): M_{G S P M}$. Masses for experimental resonances, $M_{P D G}$, have been taken from [2]. Masses from the Cornell potential, $M_{C o r}$, are also shown for comparison.

$\left(D^{*}(2007)^{0}{\overline{D^{*}}}^{0}(2007)\right.$ and $\left.D_{s}^{*+} D_{s}^{*-}\right)$ the central potential used does not make any difference for $J=0$ and $J=2$. The other new resonance that we shall call $C(4017)$ would be assigned to the $1^{++}\left(1 p_{\left[T_{1}, T_{2}\right]}\right)$ GSPM state at $4017.3 \mathrm{MeV}$. As shown above this resonance is generated altogether with $X(3872)$ as an effect of the introduction of the $D^{0}{\overline{D^{*}}}^{0}(2007)$ threshold. Hence the existence of $C(4017)$ seems to be unavoidable if the mechanism proposed for the generation of $X(3872)$ is the correct one.

For the sake of completeness let us mention that for energies quite below the first thresholds the calculated spectrum is of Cornell type giving rise to degenerate $J^{++}=$ $(0,1,2)^{++}$states. This degeneracy is broken for energies reaching the first thresholds (and beyond) due to the different values of the threshold masses in each case.

We might then conclude that an assignment of GSPM states to the existing well established or possible candidates to $0^{+}\left(J^{++}\right)$resonances is feasible. With respect to the observed differences between the calculated GSPM masses and data we shall assume that the experimental values can be reached from the GSPM ones through perturbative corrections to the hamiltonian. The experimental confirmation of the candidates and the discovery of the new predicted resonances could give definite support to this conclusion.

\subsection{2 $0\left(1^{--}\right)$GSPM States}

The spectrum for $0^{-}\left(1^{--}\right) c \bar{c}$ states from $V(r)$ up to $4200 \mathrm{MeV}$ (quite below the first threshold located at $4287 \mathrm{MeV}$, see Table 2) is shown in Table 6. The spectrum from the Cornell potential $V_{C o r}(r)$ given by (4) with the same values of the parameters $\sigma, \chi$ and $m_{c}$ is also listed for comparison.

An almost pure Cornell like spectrum (very little threshold effects) is obtained in this energy region as can be checked by comparing the calculated GSPM masses with the Cornell ones. 


\begin{tabular}{cc|cc}
$\begin{array}{c}\text { GSPM } \\
\text { States }\end{array}$ & $\begin{array}{c}M_{G S P M} \\
\mathrm{MeV}\end{array}$ & $\begin{array}{c}M_{\text {Cor }} \\
\mathrm{MeV}\end{array}$ & $\begin{array}{c}\text { Cornell } \\
\text { States }\end{array}$ \\
\hline $3 s_{\left[T_{0}, \widetilde{T}_{1}\right]}$ & 4063.2 & 4065.8 & $3 s$ \\
$2 d_{\left[T_{0}, \widetilde{T}_{1}\right]}$ & 4139.3 & 4142.8 & $2 d$ \\
$1 s_{\left[\widetilde{T}_{1}, \infty[\right.}$ & 4337.3 & & \\
$1 d_{\left[\widetilde{T}_{1}, \infty[\right.}$ & 4454.1 & 4436.5 & $4 s$ \\
$2 s_{\left[\widetilde{T}_{1}, \infty[\right.}$ & 4483.5 & 4496.1 & $3 d$
\end{tabular}

Table 7: Calculated $1^{--}$charmonium masses from 4.0 to $5.0 \mathrm{GeV}$ when only one non degenerate threshold $\widetilde{T}_{1} \equiv D^{0}{\overrightarrow{D_{1}}}^{0}(2420)$ is considered: $M_{G S P M}$. Masses from the Cornell potential, $M_{C o r}$, are also shown for comparison.

As explained before the GSPM can not be reliably applied to calculate the masses of higher spectral states in this case. Nonetheless a qualitative analysis of the possible mixing configuration content in some of the well established higher spectral resonances can be carried out. Let us centre for instance in $X(4260)$ lying close below the first (degenerate) threshold. Let us examine whether this resonance could be obtained or not if only the threshold $T_{11} \equiv D^{0}{\overline{D_{1}}}^{0}(2420)$ at $4287 \mathrm{MeV}$ were present. Then the resulting GSPM spectrum from 4.0 to $4.5 \mathrm{GeV}$ would be as listed in Table 7 where the Cornell spectrum is also given for comparison.

A glance at the table shows that the presence of the threshold would generate a new spectral state at $4337 \mathrm{MeV}$ as compared to the Cornell case. But there would not be any chance to obtain a resonance close below threshold, as the $X(4260)$. The explanation for this has to do with the fact that there is not any Cornell state close below or above threshold from which such resonance could be formed by the effect of the threshold.

The situation could change by considering the additional effect of the other degenerate threshold $T_{12} \equiv D^{0}{\overrightarrow{D_{1}}}^{0}(2430)$ on the new spectral state. As the mass of this state, $4337 \mathrm{MeV}$, is close above $T_{12}$, it could be shifted down to a value below the threshold as experimentally observed (notice that some additional attraction could also be provided by the $D^{*}(2007)^{0}{\overline{D_{0}^{*}}}^{0}(2400)$ threshold due to its large width). In this regard a refined version of the GSPM, incorporating a lesser abrupt change in the potential when approaching the threshold, could allow for a consistent treatment of the degenerate as well as the non degenerate threshold effects through the different paths followed by the potential to reach the threshold energy.

Therefore we might tentatively conclude (without any quantitative proof) that the 
existence of $X(4260)$ could be related to the presence of degenerate overlapping thresholds. Otherwise said $X(4260)$ could be the result of the mixing of the quenched $c \bar{c}$ with $D^{0}{\overline{D_{1}}}^{0}(2420)$ and $D^{0}{\overline{D_{1}}}^{0}(2430)$ configurations.

\subsubsection{Electromagnetic Widths}

Electromagnetic decay rates of charmonium are sensitive to details of the wave functions involved. Therefore their study might serve to test a quark model and to discriminate it against others. One should realize though that when ratios of decay rates are considered, similar results may be obtained from different models. Indeed some of these ratios, involving transitions from initial to final charmonium states, can be explained from heavy quark symmetry considerations without reference to any particular dynamic model [8].

Let us note that the GSPM assigns a differentiated state to each of the existing non conventional experimental candidates to be a $0^{+}\left(\mathrm{J}^{++}\right)$resonance. It also allows for an unambiguous assignment of states to conventional $0^{+}\left(\mathrm{J}^{++}\right)$and $0^{-}\left(1^{--}\right)$resonances below their first thresholds. Therefore it can be consistently used for the analysis of transitions involving these states.

We will focus on the calculation of electric dipole (E1) and two photon decay widths for which a comparative analysis to data can be carried out. Thus, for E1 decays we shall centre on transitions between spin triplet $P$ - wave and $S$ - wave states for which the non relativistic E1 partial widths read [9]

$$
\Gamma_{E 1}(i \rightarrow f+\gamma)=\frac{4 \alpha e_{c}^{2} w_{i f}^{3}\left(2 J_{f}+1\right)}{27}|\langle f|r| i\rangle|^{2}
$$

where $i$ and $f$ denote the initial (final) charmonium state, $\alpha$ stands for the fine structure constant, $e_{c}=\frac{2}{3}$ is the charm quark electric charge, $w_{i f}$ is the photon energy

$$
w_{i f}=\frac{1}{2 M_{i}}\left(M_{i}^{2}-M_{f}^{2}\right)
$$

and $\langle f|r| i\rangle$ is the dipole matrix element

$$
\langle f|r| i\rangle=\int_{0}^{\infty} R_{f}(r) r^{3} R_{i}(r) d r
$$

with $R_{f}(r)$ and $R_{i}(r)$ standing for the radial wave functions of the final and initial state respectively.

From (9) we can easily establish the ratios:

a)

$$
\frac{\Gamma_{E 1}\left(i \rightarrow f_{1}+\gamma\right)}{\Gamma_{E 1}\left(i \rightarrow f_{2}+\gamma\right)}=\frac{w_{i f_{1}}^{3}}{w_{i f_{2}}^{3}} \frac{\left|\left\langle f_{1}|r| i\right\rangle\right|^{2}}{\left|\left\langle f_{2}|r| i\right\rangle\right|^{2}}
$$


for the case in which the same initial state decays into two final $\left(f_{1}\right.$ and $\left.f_{2}\right)$ states with the same value of $J_{f}$ and

b)

$$
\frac{\Gamma_{E 1}\left(i_{1} \rightarrow f+\gamma\right)}{\Gamma_{E 1}\left(i_{2} \rightarrow f+\gamma\right)}=\frac{w_{i_{1} f}^{3}}{w_{i_{2} f}^{3}} \frac{\left|\left\langle f|r| i_{1}\right\rangle\right|^{2}}{\left|\left\langle f|r| i_{2}\right\rangle\right|^{2}}
$$

for the case in which two initial states $\left(i_{1}\right.$ and $\left.i_{2}\right)$ decay into the same final state.

As for two photon transitions we shall consider the decays from ${ }^{3} P_{0}$ and ${ }^{3} P_{2}$ states. In the nonrelativistic limit the decay widths can be expressed as [14]

$$
\begin{aligned}
& \Gamma\left(i\left({ }^{3} P_{0}\right) \rightarrow \gamma \gamma\right)=\frac{27 \alpha^{2} e_{c}^{4}}{m_{c}^{4}}\left|R_{i\left({ }^{3} P_{0}\right)}^{\prime}(0)\right|^{2} \\
& \Gamma\left(i\left({ }^{3} P_{2}\right) \rightarrow \gamma \gamma\right)=\frac{36 \alpha^{2} e_{c}^{4}}{5 m_{c}^{4}}\left|R_{i\left({ }^{3} P_{2}\right)}^{\prime}(0)\right|^{2}
\end{aligned}
$$

where $R_{i}^{\prime}(0)$ stands for the derivative of the radial wave function at the origin.

First order QCD radiative corrections to (14) and (15), in the form of multiplying factors, have been calculated. For the effective value of $\alpha_{s}=0.38$ we are using they are significant. This poses the need to calculate them to higher order. Instead we shall keep the zeroth order expressions to get a first approach to data and we shall use for practical purposes the ratios

$$
\begin{aligned}
& \frac{\Gamma\left(i_{2}\left({ }^{3} P_{0}\right) \rightarrow \gamma \gamma\right)}{\Gamma\left(i_{1}\left({ }^{3} P_{0}\right) \rightarrow \gamma \gamma\right)}=\frac{\left|R_{i_{2}\left({ }^{3} P_{0}\right)}^{\prime}(0)\right|^{2}}{\left|R_{i_{1}\left({ }^{3} P_{0}\right)}^{\prime}(0)\right|^{2}} \\
& \frac{\Gamma\left(i_{2}\left({ }^{3} P_{2}\right) \rightarrow \gamma \gamma\right)}{\Gamma\left(i_{1}\left({ }^{3} P_{2}\right) \rightarrow \gamma \gamma\right)}=\frac{\left|R_{i_{2}\left({ }^{3} P_{2}\right)}^{\prime}(0)\right|^{2}}{\left|R_{i_{1}\left({ }^{3} P_{2}\right)}^{\prime}(0)\right|^{2}}
\end{aligned}
$$

where the multiplying factors cancel out.

$$
\chi_{c J}(\mathbf{1 P})
$$

The $\chi_{c J}(1 P)$ resonances are identified with the $J^{++}\left(1 p_{\left[T_{0}, T_{1}\right]}\right)$ GSPM states which are practically identical to the $J^{++}(1 p)$ Cornell states. As the $J / \psi$ description is also the same with both models they give the same results for the $\chi_{c J}(1 P) \rightarrow \gamma J / \psi$ decay widths if the same values for the photon energies are chosen.

The calculated GSPM widths are shown in Table 8 where the dipole matrix elements are also tabulated. For the photon energies the experimental values have been used. This can be justified under our former assumption that the experimental masses can be reached from the GSPM ones through first order perturbative corrections to the hamiltonian (let us remind that no modification of the wave functions is then generated). 


\begin{tabular}{|c|c|c|c|c|c|}
\hline$f$ & $i$ & $\begin{array}{l}\left(w_{i f}\right)_{\operatorname{Exp}} \\
\mathrm{MeV}\end{array}$ & $\underset{\mathrm{fm}^{2}}{|\langle f|r| i\rangle|^{2}}$ & $\begin{array}{c}\left(\Gamma_{E 1}(i \rightarrow f+\gamma)\right)_{G S P M} \\
\mathrm{MeV}\end{array}$ & $\begin{array}{c}\left(\Gamma_{E 1}(i \rightarrow f+\gamma)\right)_{\text {Exp }} \\
\mathrm{MeV}\end{array}$ \\
\hline \multirow[t]{3}{*}{$J / \psi$} & $\chi_{c 0}(1 P)$ & 303.04 & 0.198 & 0.20 & $0.13 \pm 0.02$ \\
\hline & $\chi_{c 1}(1 P)$ & 389.36 & 0.198 & 0.43 & $0.29 \pm 0.02$ \\
\hline & $\chi_{c 2}(1 P)$ & 429.63 & 0.198 & 0.58 & $0.37 \pm 0.04$ \\
\hline
\end{tabular}

Table 8: Calculated E1 dipole matrix elements (fourth column) and decay widths (fifth column) for $\chi_{c J}(1 P) \rightarrow \gamma J / \psi$. Photon energies (third column) from (10) with experimental masses [2]. Widths data (sixth column) from [2].

As can be checked the values obtained are $30 \%$ off the experimental intervals. This can be considered a reasonable first approach to data and a starting point to include additional corrections (see [13] and references therein). Although we do not proceed here along this line it is worth to point out that the central values of the experimental ratios

$$
\frac{\left(\Gamma_{E 1}\left(\chi_{c 2}(1 P) \rightarrow \gamma J / \psi\right)\right)_{E x p}}{\left(\Gamma_{E 1}\left(\chi_{c 0}(1 P) \rightarrow \gamma J / \psi\right)\right)_{E x p}}=2.9 \pm 0.8
$$

and

$$
\frac{\left(\Gamma_{E 1}\left(\chi_{c 1}(1 P) \rightarrow \gamma J / \psi\right)\right)_{E x p}}{\left(\Gamma_{E 1}\left(\chi_{c 0}(1 P) \rightarrow \gamma J / \psi\right)\right)_{E x p}}=2.2 \pm 0.6
$$

are in good agreement with the experimental photon energy ratios

$$
\begin{aligned}
& \frac{\left(w_{\chi_{c 2(1 P) J / \psi}}^{3}\right)_{E x p}}{\left(w_{\chi_{c 0(1 P) J / \psi}}^{3}\right)_{E x p}}=2.9 \\
& \frac{\left(w_{\chi_{c 2(1 P) J / \psi}}^{3}\right)_{E x p}}{\left(w_{\chi_{c 1(1 P) J / \psi}}^{3}\right)_{E x p}}=2.1
\end{aligned}
$$

Taking into account (13) this suggests the additional corrections should not introduce any significant difference among the $\chi_{c 0}(1 P), \chi_{c 1}(1 P)$ and $\chi_{c 2}(1 P)$ wave functions.

Regarding two photon decay widths, the degeneracy of the $J^{++}\left(1 p_{\left[T_{0}, T_{1}\right]}\right)$ GSPM states gives rise from (14) and (15) to the ratio

$$
\frac{\left(\Gamma\left(\chi_{c 0}(1 P) \rightarrow \gamma \gamma\right)\right)_{G S P M}}{\left(\Gamma\left(\chi_{c 2}(1 P) \rightarrow \gamma \gamma\right)\right)_{G S P M}}=\frac{27}{\left(\frac{36}{5}\right)}=3.75
$$




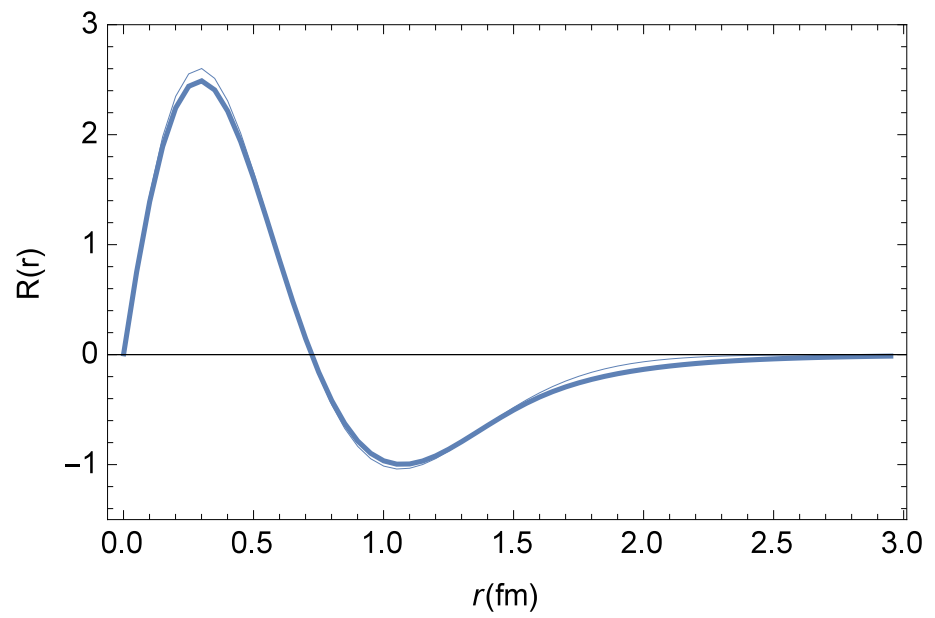

Figure 3: Radial wave functions R(r) (in units $\left.f m^{-\frac{3}{2}}\right)$ for the $2^{++}\left(2 p_{\left[T_{0}, T_{1}\right]}\right)$ GSPM state (thick line) and the $2^{++}(2 p)$ Cornell state (thin line).

within the experimental interval [2]

$$
\frac{\left(\Gamma\left(\chi_{c 0}(1 P) \rightarrow \gamma \gamma\right)\right)_{E x p}}{\left(\Gamma\left(\chi_{c 2}(1 P) \rightarrow \gamma \gamma\right)\right)_{E x p}}=\frac{2.3 \pm 0.4 \mathrm{KeV}}{0.53 \pm 0.07 \mathrm{KeV}}=4.3 \pm 1.6
$$

$\chi_{c 2}(\mathbf{2 P})$

The $\chi_{c 2}(2 P)$ resonance at $3927 \mathrm{MeV}$ is assigned to the $2^{++}\left(2 p_{\left[T_{0}, T_{1}\right]}\right)$ GSPM state that differs little from the $2^{++}(2 p)$ Cornell one, as shown in Fig. 3 .

Only an experimental lower bound for the two photon decay width is known [2]

$$
\left(\Gamma\left(\chi_{c 2}(2 p) \rightarrow \gamma \gamma\right)\right)_{E x p}>0.21 \pm 0.04 \mathrm{eV}
$$

from

$$
\left(\Gamma\left(\chi_{c 2}(2 p) \rightarrow \gamma \gamma\right) \mathcal{B}\left(\chi_{c 2}(2 p) \rightarrow D \bar{D}\right)\right)_{E x p}=0.21 \pm 0.04 \mathrm{eV}
$$

where $\mathcal{B}$ stands for branching fraction.

From the calculated GSPM wave functions we get from (17) the ratio

$$
\frac{\left(\Gamma\left(\chi_{c 2}(2 p) \rightarrow \gamma \gamma\right)\right)_{G S P M}}{\left(\Gamma\left(\chi_{c 2}(1 p) \rightarrow \gamma \gamma\right)\right)_{G S P M}}=\frac{\left(\left|R_{2^{++}\left(2 p_{\left[T_{0}, T_{1}\right]}\right)}^{\prime}(0)\right|^{2}\right)_{G S P M}}{\left(\left|R_{2^{++}\left(1 p_{\left[T_{0}, T_{1}\right]}^{\prime}\right)}^{\prime}(0)\right|^{2}\right)_{G S P M}}=1.34
$$


By assuming that this value is a reasonable approach to the experimental ratio we might expect the approximated values

$$
\begin{gathered}
\Gamma\left(\chi_{c 2}(2 p) \rightarrow \gamma \gamma\right) \simeq 1.34\left(\Gamma\left(\chi_{c 2}(1 p) \rightarrow \gamma \gamma\right)\right)_{E x p}=0.71 \pm 0.09 \mathrm{keV} \\
\mathcal{B}\left(\chi_{c 2}(2 p) \rightarrow D \bar{D}\right) \simeq 0.30 \pm 0.10
\end{gathered}
$$

\section{$\mathrm{X}(3872)$}

As shown before the $1^{++}\left(2 p_{\left[T_{0}, T_{1}\right]}\right)$ GSPM state is identified with the $X(3872)$ whose mass has been used to fine tune the charm quark mass.

Concerning electromagnetic decays the ratio

$$
A \equiv \frac{\Gamma(X(3872) \rightarrow \gamma \psi(2 s))}{\Gamma(X(3872) \rightarrow \gamma J / \psi)}
$$

has been recently measured [15] to be

$$
A_{\text {Exp }}=2.46 \pm 0.64 \pm 0.29
$$

compatible with the previous value $3.4 \pm 1.4$ [16] and the upper bound $<2.1$ [17].

From (12) the GSPM gives for this ratio the value

$$
A_{G S P M}=2.01
$$

calculated from the dipole matrix elements

$$
\begin{gathered}
\left|\langle\psi(2 s)|r| X(3872)\rangle_{G S P M}\right|^{2}=0.2856 \mathrm{fm}^{2} \\
\left|\langle J / \psi|r| X(3872)\rangle_{G S P M}\right|^{2}=0.0025 \mathrm{fm}^{2}
\end{gathered}
$$

and the experimental values of the photon energies

$$
\begin{aligned}
& \left(w_{X(3872) \psi(2 s)}\right)_{E x p}=181.25 \mathrm{MeV} \\
& \left(w_{X(3872) J / \psi}\right)_{E x p}=697.19 \mathrm{MeV}
\end{aligned}
$$

Therefore a full compatibility with existing data comes out. We should point out though that quite the same result would be obtained for the dipole matrix elements by using the $1^{++}(2 p)$ Cornell state wave function instead of the $1^{++}\left(2 p_{\left[T_{0}, T_{1}\right]}\right)$ GSPM one. As the main difference between these two wave functions is the long tail of $2 p_{\left[T_{0}, T_{1}\right]}$ state as compared to that of $2 p$ (see Fig. 2) we may conclude that these radiative decays are not sensitive to the long distance nature of $X(3872)$. This can be understood by the negligible long distance overlap of the $1^{++}\left(2 p_{\left[T_{0}, T_{1}\right]}\right)$ state with $J / \psi$ and $\psi(2 s)$. (The calculated root mean square radii for $J / \psi$ and $\psi(2 s)$ are respectively $0.5 \mathrm{fm}$ and $0.9 \mathrm{fm}$.) It should be mentioned that the same conclusion has been also inferred by other authors using molecular descriptions for $X(3872)$ [18, 19]. 


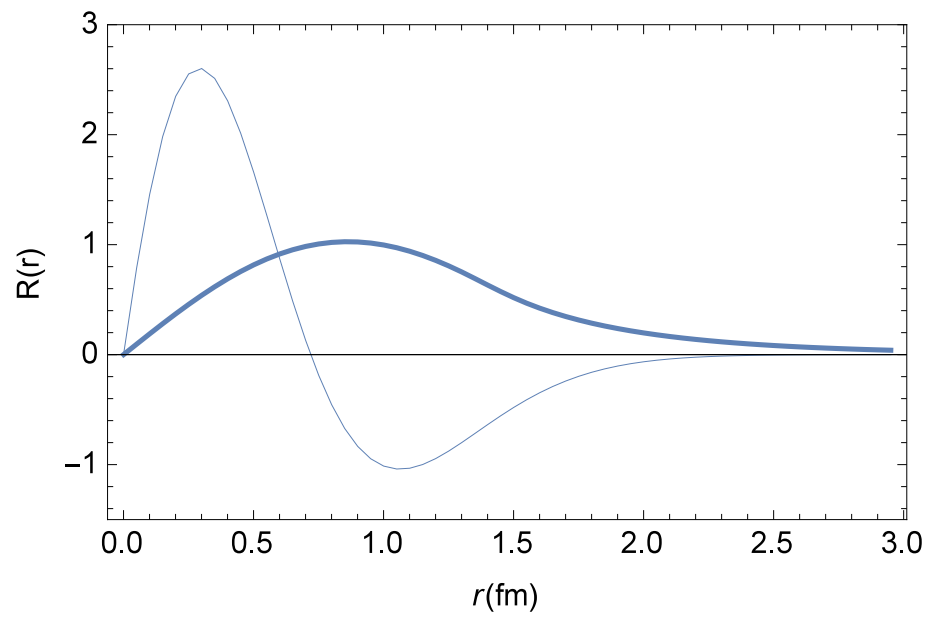

Figure 4: Radial wave functions R(r) (in units $\mathrm{fm}^{-\frac{3}{2}}$ ) for the $0^{++}\left(1 p_{\left[T_{0}, T_{1}\right]}\right)$ GSPM state (thick line) and the $0^{++}(2 p)$ Cornell state (thin line).

\section{$\mathrm{X}(3915)$}

The Review of Particle Properties 2] has identified the $X(3915)$ with a conventional $\chi_{c 0}(2 p)$, this is with a $0^{++}(2 p)$ Cornell like state. This identification has been criticized by some authors [20, 21]. A major criticism is the lack of evidence of $X(3915) \rightarrow D \bar{D}$ decays. From our estimation above for the branching fraction $\mathcal{B}\left(\chi_{c 2}(2 p) \rightarrow D \bar{D}\right) \simeq 0.30$ a similar result could be expected for $\mathcal{B}\left(\chi_{c 0}(2 p) \rightarrow D \bar{D}\right)$ since the $0^{++}(2 p)$ and $2^{++}(2 p)$ Cornell states are degenerate and the measured values of the masses and total widths of $\chi_{c 2}(2 p)$ and $X(3915)$ are quite similar. [2]

Regarding electromagnetic processes a lower bound for the two photon decay width

$$
(\Gamma(X(3915) \rightarrow \gamma \gamma))_{E x p}>54 \pm 9 \mathrm{eV}
$$

is known from

$$
(\Gamma(X(3915) \rightarrow \gamma \gamma) \mathcal{B}(X(3915) \rightarrow J / \psi \omega))_{E x p}>54 \pm 9 \mathrm{eV}
$$

The GSPM assigns the $X(3915)$ to the $0^{++}\left(1 p_{\left[T_{1}, T_{2}\right]}\right)$ state that differs greatly from the $0^{++}(2 p)$ Cornell one as shown in Fig. 4 .

From the calculated GSPM wave functions we get from (16) the ratio

$$
\frac{(\Gamma(X(3915) \rightarrow \gamma \gamma))_{G S P M}}{\left(\Gamma\left(\chi_{c 0}(1 P) \rightarrow \gamma \gamma\right)\right)_{G S P M}}=\frac{\left|R_{0^{++}\left(1 p_{\left[T_{1}, T_{2}\right]}\right)}^{\prime}(0)\right|^{2}}{\left|R_{0^{++}\left(1 p_{\left[T_{0}, T_{1}\right]}\right)}^{\prime}(0)\right|^{2}}=0.02
$$


Assuming again that this value is a reasonable approach to the experimental ratio we might expect

$$
\Gamma(X(3915) \rightarrow \gamma \gamma) \simeq 0.02\left(\Gamma\left(\chi_{c 0}(1 p) \rightarrow \gamma \gamma\right)\right)_{E x p}=44 \pm 7 \mathrm{eV}
$$

By combining this result with the experimental lower bound given above we would get

$$
\begin{gathered}
\Gamma(X(3915) \rightarrow \gamma \gamma) \simeq 48 \pm 3 \mathrm{eV} \\
\mathcal{B}(X(3915) \rightarrow J / \psi \omega)>0.88
\end{gathered}
$$

It should be emphasized that the identification of $X(3915)$ with $0^{++}(2 p)$ would give a completely different ratio

$$
\frac{(\Gamma(X(3915) \rightarrow \gamma \gamma))_{\text {Cornell }}}{\left(\Gamma\left(\chi_{c 0}(1 P) \rightarrow \gamma \gamma\right)\right)_{\text {Cornell }}}=\frac{\left|R_{0^{++}(2 p)}^{\prime}(0)\right|^{2}}{\left|R_{0^{++}(1 p)}^{\prime}(0)\right|^{2}}=1.2
$$

and consequently completely different values for the two photon decay width $(2640 \mathrm{eV})$ and the branching fraction to $J / \psi \omega(>0.02)$.

Certainly the big GSPM branching fraction for $X(3915) \rightarrow J / \psi \omega$, OZI suppressed in the Cornell model, should be somehow justified. A quantitative justification, if possible, would imply the development of a strong decay theory above threshold within the GSPM framework which is outside the scope of this work. Hence we shall limit here to a merely speculative qualitative comment. Let us imagine for instance that due to the threshold modulation the GSPM interaction favored, at the energy of $X(3915)$ and through light quark pair creation out of the vacuum, the formation of color octets made of heavy quark-light antiquark and viceversa. Then it would be possible to have a dominant decay through reordering of the quarks in such a state like $J / \psi \omega$. On the contrary the Cornell states are known to favor the formation of color singlets giving rise to the dominant fall apart decay mode $D \bar{D}$.

Therefore the GSPM and Cornell descriptions represent incompatible scenarios for the understanding of $X(3915)$. According to our analysis more detailed data could defi-

nitely clarify the situation about the true nature (non conventional or conventional) of this resonance.

\section{Summary}

A nonrelativistic quark model called Generalized Screened Potential Model, or abbreviate GSPM, previously used to calculate the bottomonium spectrum has been applied to charmonium. 
The model, whose interaction potential has a Cornell form but modulated by mesonmeson thresholds, has been used to calculate $0^{+}\left(\mathrm{J}^{++}\right)$charmonium masses up to $4.4 \mathrm{GeV}$, a limit imposed to the application of the model by the incomplete current knowledge of open charm meson-meson thresholds. As it turned out to be the case in bottomonium a richer spectrum (bigger number of bound states) than the one resulting from the nonscreened Cornell potential is predicted. However, differing from bottomonium where the lack of data prevented the verification or refutationof such a prediction, there exist in charmonium well established as well as candidates to non conventional resonances in the energy interval analyzed. As a matter of fact the well established $X(3872)$ is nicely described as a GSPM state that can be interpreted as being generated from the $D^{0}{\overline{D^{*}}}^{0}(2007)$ threshold and the $1^{++}(2 p)$ Cornell state. Regarding the experimental candidates $X(4140) 0^{+}\left(?^{?+}\right)$ and $X(4350) 0^{+}\left(?^{?+}\right)$ a good spectral correspondence with GSPM states is observed. Furthermore two new $0^{+}\left(\mathrm{J}^{++}\right)$resonances are predicted, a $0^{+}\left(2^{++}\right)$or $0^{+}\left(0^{++}\right)$one with mass around $4140 \mathrm{MeV}$ and a $0^{+}\left(1^{++}\right)$one with mass around $4017 \mathrm{MeV}$. (Notice though that all the calculated GSPM masses, except for $X(3872)$ which is used to fine tune the quark mass, are below the experimental ones what suggests that the masses of these new resonances could also be underestimated.) The generation of these new resonances in the GSPM is related to the presence of the states assigned to $X(4140)$ and $X(3872)$. Therefore their discovery would constitute a definite check of the GSPM as a model for the spectral description.

The GSPM has also been employed to evaluate the $0^{-}\left(1^{--}\right)$spectrum up to $4.2 \mathrm{GeV}$, a limit imposed to the applicability of the model by the presence of degenerate and overlapping thresholds. The resulting spectrum in this energy region is very much of Cornell type. In order to go further in energy the model should be refined. With respect to this a simplified qualitative analysis of the possible generation of $X$ (4260) seems to point out that the joint effect from overlapping meson-meson configurations should be an essential ingredient to be incorporated.

A study of electromagnetic decays of $0^{+}\left(\mathrm{J}^{++}\right)$resonances, specifically E1 and two photon decays for which there are data available, has also been carried out. The calculated GSPM widths are fully compatible with existing data. However, more detailed data are needed to perform a stringent check of the GSPM. In this regard a thorough experimental analysis of $X(3915)$ is of particular interest given the very different description coming out from the GSPM and the Cornell models.

This work has been supported by Ministerio de Economía y Competitividad of Spain (MINECO) grant FPA2013-47443-C2-1-P, and by PrometeoII/2014/066 from Generalitat Valenciana.

\section{References}

[1] P. González. J.Phys. G 41, 095001 (2014); arXiv:1406.5025 [hep-ph]. 
[2] K. A. Olive et al. [Particle Data Group (PDG)], Chin. Phys. C 38, 090001 (2014).

[3] S. L. Olsen, Front.Phys. 10, 121 (2015); arXiv:1411.7738 [hep-ex].

[4] N. Brambilla et al., Eur. Phys. J. C 71, 1534 (2011).

[5] G. S. Bali, H. Neff, T. Düssel, T. Lippert and K. Schilling (SESAM Collaboration), Phys. Rev. D 71, 114513 (2005).

[6] B-Q. Li and K-T. Chao, Phys. Rev. D 79, 094004 (2009).

[7] P. González, Phys. Rev. D 80, 054010 (2009).

[8] P. Cho and M. B. Wise, Phys. Lett. B 346, 129 (1995).

[9] E. Eichten, K. Gottfried, T. Kinoshita, K. D. Lane and T. M. Yan, Phys. Rev. D 21, 203 (1980).

[10] S. Godfrey and N. Isgur, Phys. Rev. D 32, 189 (1985).

[11] G. S. Bali, Phys. Rep. 343, 1 (2001).

[12] A. M. Badalian and V. L. Morgunov, Phys. Rev. D 60, 116008 (1999).

[13] E. Eichten, S. Godfrey, H. Mahlke, and J. L. Rosner, Rev. Mod. Phys. 80, 1161 (2008).

[14] W. Kwong, P. B. Mackenzie, R. Rosenfeld and J. L. Rosner, Phys. Rev. D 37, 3210 (1988).

[15] R. Aaij et al. (LHCb Collaboration), Nucl. Phys. B 886, 665 (2014).

[16] B. Aubert et al. (BaBar Collaboration), Phys. Rev. Lett 102, 132001 (2009).

[17] V. Bhardwaj et al. (Belle Collaboration), Phys. Rev. Lett 107, 091803 (2011).

[18] T. Mehen, R. Springer, Phys. Rev. D 83, 094009 (2011).

[19] F. K. Guo, C. Hanhart, Yu.S. Kalashnikova, U. G. Meissner and A. V. Nefediev, Phys. Lett. B 742, 394 (2015).

[20] F. K. Guo and U. G. Meissner, Phys. Rev. D 86, 091501 (2012).

[21] S. L. Olsen, Phys. Rev. D 91, 057501 (2015); arXiv:1410.6534 [hep-ex]. 